\title{
Deep Vein Thrombosis Prophylaxis Evaluation in Intensive Care Unit
}

\author{
Banafsheh Nekoonam, ${ }^{1}$ Azadeh Eshraghi, ${ }^{2}$ Mohammadreza Hajiesmaeili, ${ }^{3}$ and Zahra Sahraei ${ }^{4,}{ }^{*}$ \\ ${ }^{1}$ Student Research Committee, School of Pharmacy, Shahid Beheshti University of Medical Sciences, Tehran, IR Iran \\ ${ }^{2}$ Department of Clinical Pharmacy, Faculty of Pharmacy-International Campus, Iran University of Medical Sciences, Tehran, IR Iran \\ ${ }^{3}$ Anesthesiology Research Center, Anesthesia and Critical Care Department, Loghman Hakim Hospital, Shahid Beheshti University of Medical Sciences, Tehran, IR Iran \\ ${ }^{4}$ Department of Clinical Pharmacy, School of Pharmacy, Shahid Beheshti University of Medical Sciences, Tehran, IR Iran \\ "Corresponding author: Zahra Sahraei, Department of Clinical Pharmacy, School of Pharmacy, Shahid Beheshti University of Medical Sciences, Tehran, IR Iran. Tel: +98-21 \\ 88200209, Fax: +98-21 88873704, E-mail: zahra.sahraee@yahoo.com
}

Received 2015 September 30; Accepted 2015 November 14.

\begin{abstract}
Background: One of the main causes of morbidity in hospitalized patients, and especially in the intensive care unit (ICU), is venous thromboembolism (VTE). The best way to deal with VTE is prophylaxis. Rational prophylaxis should be provided after risk factor analysis. Different prophylaxis regimens are pharmacological and physical. Pharmacological prophylaxis regimens consist of heparin and enoxaparin that are given subcutaneously.

Objectives: The current study aimed to investigate appropriate administration of deep vein thrombosis (DVT) prophylaxis in ICU in an educational hospital.

Methods: Caprini risk assessment model was employed to evaluate patients' risk factors. Immobile patients (for three days) with at least one thrombotic risk factor were selected. The Caprini scores were identified from patients' medical record. Three groups were identified after obtaining the scores: patients at low, medium and high risks. For each group, appropriate prophylaxis regimen was recommended.

Results: A total of 52 patients were evaluated, about $88.46 \%$ of the subjects had DVT prophylaxis indication; $10.71 \%$ received physical prophylaxis and $89.29 \%$ of them received pharmacological prophylaxis. For two-thirds of the patients with pharmacological prophylaxis enoxaparin was administered and for the other part heparin. Totally, $32.61 \%$ of the subjects received correct prophylaxis, $17.39 \%$ received inadequate prophylactic doses and $6.52 \%$ received higher doses. Totally, $67.39 \%$ of the subjects did not receive correct prophylaxis.

Conclusions: According to the collected data, appropriate DVT prophylaxis was not prescribed. It is required to pay serious attention to this medication error. It is suggested to invest on educating medical teams about DVT prophylaxis, which is highly demanded.
\end{abstract}

Keywords: Deep Vein Thrombosis Prophylaxis, Caprini Risk Assessment Model, Risk Score, Intensive Care Unit

\section{Background}

Annually, about 2 million people experience a deep vein thrombosis (DVT) and about 600,000 of them experience a pulmonary embolism (PE) that each year causes death in approximately 200,000 patients (1). For patients in surgical and obstetrics departments and intensive care units (ICUs), one of the significant morbidity reasons is venous thromboembolism (VTE). About 129.90-395.16 cases per 1000 Iranian adult inpatients with the risk of DVT experienced DVT annually (2).

VTE mainly consists of deep vein thrombosis (DVT) and pulmonary embolism (PE). Clinical assessment of DVT or $P E$ is not simple and the results are not reliable unless specific symptoms are observed. Approximately 65\% of DVT cases show no specific symptoms and PE is frequently asymptomatic. Therefore, adequate VTE prophylaxis is needed to prevent DVT and PE and their morbidity. Appro- priate prophylaxis can be provided by risk factor analysis (RFA) (3). One of the best and easiest methods of RFA is scoring system (4). RFA is needed due to prophylaxis risk such as bleeding or heparin-induced thrombocytopenia. After RFA assessment, patients who underwent surgery are divided into different groups. For patients at moderate to high risk, pharmacologic prophylaxis treatment should be prescribed although pharmacologic prophylaxis for patients at low risk may not be necessary after risk benefit evaluation (3). It should be considered that DVT or PE may happen up to several months after surgery or major trauma (5).

According to the guidelines for prevention of VTE of American college of chest physicians (ACCP) in 2008, there is at least one risk factor for VTE in about all hospitalized patients and nearly $40 \%$ of them show three risk factors or more. Cancer, trauma, history of VTE and major orthopedic procedures are considered as high risk states 
(6) that nearly all ICU patients have at least one risk factor for DVT or PE, and additional risk factors are supposed to have a cumulative effect according to American society of health-system pharmacists. One of the most common and serious complications in ICU patients was venous thromboembolism and PE causes about 10\% of hospital deaths (7). In comparison with VTE treatment, prophylaxis has more significant effect and is more cost-effective (8). Thus an adequate prophylaxis (medicinal or physical) is necessary for ICU patients. VTE prophylaxis includes mechanical and pharmacological prophylaxis. Mechanical prophylaxis consists of graduated compression stockings (GCS) and intermittent pneumatic compression (IPC) devices used for patients at low risk. Subcutaneous unfractionated heparin (UFH) and low molecular weight heparin (LMWH) are common pharmacological prophylaxis regimens that are usually prescribed (9).

The importance of inattention to deep vein thrombosis can be interpreted from recurrent episodes of DVT or PE that is called postphlebitic syndrome (PTS) presents by a range of symptoms; leg edema, pain, aching and tiredness, skin discoloration, scarring, and even open ulceration (3). A survey conducted in 2015 in Iran showed that VTE causes lots of morbidities in hospitals. Numerous cases of VTE occur due to inadequate DVT prophylaxis (2).

Patients admitted to ICUs, due to immobilization and usually high thrombotic risk factors, are at the highest risk of DVT. Therefore, an adequate prophylaxis (medicinal or physical) is necessary for them.

\section{Objectives}

The current study aimed to evaluate proper administration of DVT prophylaxis in patients admitted to ICU by Caprini risk assessment model.

\section{Methods}

Patients in General ICU of Loghman Hakim educational hospital, Tehran, Iran, were investigated for DVT risk factors. This study was conducted from June to September 2015. Based on the literature which proves the validity of Caprini risk assessment model, this model was used to evaluate patients' risk scores to define risk category (10).

VTE prophylaxis was considered in medical patients with limited mobility for three days (at least $50 \%$ of times on bed), and at least one of the by thrombotic risk factors. Using the check list presented in Table 1, patients' VTE risk assessment was performed by a pharmacist who attended in ICU at least five hours per day per week.

Patients were excluded if pharmacologic DVT prophylaxis was contraindicated such as: active major bleeding, coagulopathy (platelets $<50,000$ or international normalized ratio (INR) $>1.5$, recent head trauma, bleeding risk due to epidural catheter.

When the risk points were identified, correct prophylaxis regimens were evaluated:

- Low risk ( $\leq 1$ total risk point)

Early ambulation:

Out of bed to chair

Out of bed with assistance

Sequential compression device (SCD)

Plexi-pulse

- Moderate risk (2 total risk points)

Heparin 5000 U q subcutaneous (SQ)12 hours

Heparin 5000 U q SQ 8 hours

Enoxaparin $40 \mathrm{mg}$ SQ q 24 hours

Enoxaparin $30 \mathrm{mg}$ SQ q 24 hours (if CLcr $<30 \mathrm{~mL} / \mathrm{min}$ )

SCD

Plexi-pulse

- High risk ( $\geq 3$ total risk points)

Heparin 5000 U q SQ 8 hours

Enoxaparin $40 \mathrm{mg}$ SQ q 24 hours

Enoxaparin 30 mg SQ q 12 hours (Orthopedic surgery, major trauma and spinal cord injury)

Enoxaparin $30 \mathrm{mg}$ SQ q 24 hours (if CLcr $<30 \mathrm{~mL} / \mathrm{min}$ )

In addition to the above (optional):

SCD

Elastic stocking

Plexi-pulse

When bleeding risk precludes the use of anticoagulants for DVT prophylaxis, one of the following actions should be considered: SCD, elastic stocking + SCD, elastic stocking, Plexi-pulse.

The data were analyzed using Microsoft excel and SPSS version 21.0. The results are expressed in terms of numbers and percentages.

\section{Results}

Totally, 52 patients were evaluated, 33 males (63.46\%) and 19 females (36.54\%). The patients' age range was 5 90 with the mean \pm SD of $43.02 \pm 24.63$. Nearly $88.46 \%$ of the subjects had indication for DVT prophylaxis and about $11.54 \%$ were not included due to anticoagulants contraindication. No patient received anticoagulation due to no need for their administration.

Among the patients with indication of DVT prophylaxis, $60.87 \%$ received prophylaxis and $39.13 \%$ did not receive it. Among the latter group, 66.67\% did not receive any prophylaxis and 33.33\% had contraindication for pharmacological prophylaxis but did not experience physical prophylaxis (the used physical prophylaxis was leg bandages). 
Table 1. Scoring Check List

\begin{tabular}{|c|c|c|}
\hline Risk Factors Score 1 & Risk Factors Score 2 & Risk Factors Score 3 \\
\hline Patient's age 40 - $60 \mathrm{y} / 0$ & Patients $>60$ years old & Multiple trauma (present) \\
\hline Smoker (present) & Documented history of DVT / PE & Chemotherapy (current course) \\
\hline Family history of DVT/PE & Acute heart failure & Cancer (past and present) \\
\hline History of heart failure & & Inherited thrombophilia $^{a}$ \\
\hline Ischemic stroke (past and present) & & Antiphospholipid antibody syndrome \\
\hline $\begin{array}{l}\text { Leg swelling, ulcers, stasis, peripheral vascular diseases, } \\
\text { varicose veins and vacuities }\end{array}$ & & $\begin{array}{l}\text { Myeloproliferative disorders (polycythemia vera, essential } \\
\text { thrombocythemia) }\end{array}$ \\
\hline Acute infections including pneumonia & & Paroxysmal nocturnal hemoglobinuria \\
\hline $\operatorname{Obesity}(>120 \%$ IBW $)$ & & Orthopedic surgery ( below waist) \\
\hline $\begin{array}{l}\text { Current use of oral contraceptives, estrogen therapy, } \\
\text { Hormone replacement therapy or drugs such as } \\
\text { tamoxifen, thalidomide and lenalidomide }\end{array}$ & & Pelvic / thoracic / abdominal surgery ( $\leq 1$ month) \\
\hline Nephrotic syndrome & & Spinal cord injury ( $\leq 1$ month) or paraplegia \\
\hline Pregnancy or postpartum ( $<1$ month) & & Surgery lasting $>30$ minutes \\
\hline Indwelling central venous catheter(present) & & Sepsis \\
\hline \multicolumn{3}{|l|}{ Sickle cell disease } \\
\hline Immobility & & \\
\hline
\end{tabular}

Prophylaxis was pharmacological or physical with the incidence shown in Figure 1.

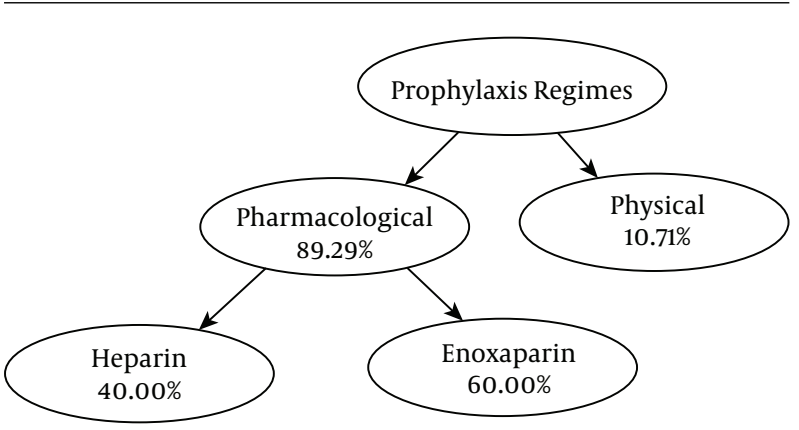

Figure 1. DVT Prophylaxis Regimens

The enoxaparin and heparin administration patterns for DVT prophylaxis are shown in Figures 2 and 3, respectively; $73.08 \%$ of all patients were at high risk due to having 3 risk points or more; $11.54 \%$ had 2 risk points (moderate risk) and $15.38 \%$ had 1 risk point or less (low risk).

The highest scores were given to immobility $(\mathrm{n}=30 \mathrm{pa}$ tients), surgery lasting more than 30 minutes ( $\mathrm{n}=28 \mathrm{pa}$ tients) and leg swelling ( $\mathrm{n}=25$ patients).

Preexisting diseases of the patients that caused hospitalization were in the following order: tumor (38.46\%), accident (13.64\%), cerebral aneurysm (5.77\%), hydrocephalus (5.77\%), abdominal surgery (3.85\%) and cholangitis (1.92\%), ileus, subdural hematoma, cranioplasty, acute renal failure, necrotizing fasciitis, refractory seizure, pituitary ade-

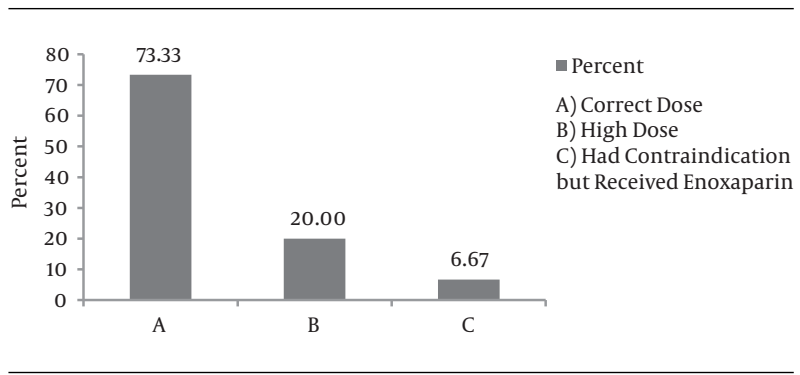

Figure 2. Enoxaparin Administration Pattern

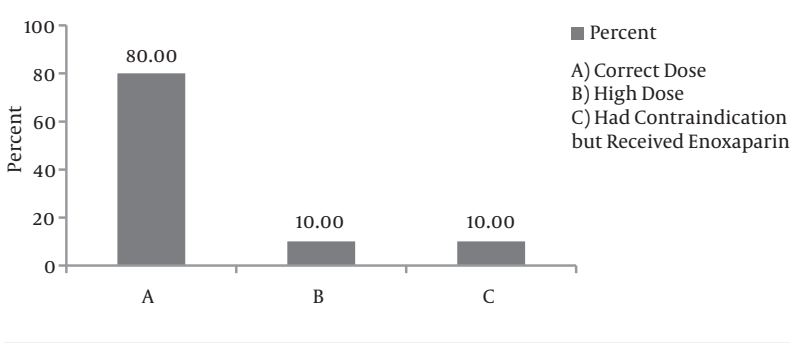

Figure 3. Heparin Administration Pattern

noma, acute abdomen, etc.

\section{Discussion}

The obtained data showed that $67.39 \%$ of the subjects with indication of DVT prophylaxis did not receive correct prophylaxis. The most frequent errors were inadequate 
heparin doses (about 17.39\%) and not considering DVT prophylaxis for $39.13 \%$ of the subjects. Therefore, there was no acceptable process for DVT prophylaxis. The study results were in accordance with Sharif-Kashani et al. reported that appropriate prophylaxis was provided for less than half of the patients in need (2).

Zeitoun et al., concluded that the need for implementing proved guidelines was needed to improve the overall patient safety. They recorded that inappropriate VTE prophylaxis was administered in $35 \%$ of the subjects in lowrisk group, $70 \%$ of the subjects in the moderate-risk group, and $39 \%$ of the subjects in the high-risk group $(\mathrm{P}<0.0001)$ (11). In the current study the prophylaxis was inadequate in $83.33 \%$ of moderate and $65.79 \%$ of high risk group subjects.

Another study conducted in London in 2007, showed that $16 \%$ of the administered enoxaparin doses required adjustments (12). In the current study $20 \%$ of the prescribed enoxaparin doses needed adjustment. In addition, $6.67 \%$ of the subjects received enoxaparin but they had contraindication; therefore contraindications should be considered too. Therefore, totally $26.67 \%$ of the prescribed enoxaparin were not correct.

The study by Fahimi et al. in Masih Hospital in Tehran, Iran, reviewed enoxaparin utilization on 147 inpatients. Their results showed that inappropriate dosing, administration and prescription of enoxaparin was rather common in Masih Hospital and they needed educational programs and implementation of protocols to control prescription patterns (13). The current study showed that enoxaparin administration pattern was inappropriate similar to that of the study by Fahimi et al., but it was better than heparin pattern. Since only $10 \%$ of heparin doses were correct and unfortunately $80.00 \%$ received inadequate heparin doses.

Another study on pediatric patients in a tertiary medical center, showed that less than $40 \%$ of the patients were administered appropriate empirical treatment dosing of enoxaparin (14). However, in the current evaluation $73.33 \%$ of patients, who received enoxaparin, received correct doses.

Another study evaluated utilization pattern of anticoagulants and highlighted the importance of application of guidelines for appropriate use of anticoagulants (15); similar to the current study that showed more than two thirds of the subjects received improper DVT prophylaxis in the absence of guidelines. It indicates the necessity of practical guidelines for DVT risk assessment and appropriate anticoagulant prescription.

In addition, Khalili et al. evaluated utilization of anticoagulants in 400 patients from four wards (infectious disease, cardiology, nephrology and cardiac care unit) of a tertiary teaching hospital in a cross-sectional, prospec- tive study and their findings showed that 11 (2.8\%) patients had received inappropriate treatment with anticoagulants (16).

Therefore, despite the existence of standard guidelines to prevent and treat VTE, this administration was not performed accurately. Therefore, to achieve appropriate usage of anticoagulants, it is necessary to invest on educating medical teams about DVT prophylaxis. Also, it is required to pay attention to this regular medication error to decrease morbidity and mortality related to inappropriate anticoagulant use.

\section{Footnotes}

Authors' Contribution: Study concept and design: Zahra Sahraei, Mohammadreza Hajiesmaeili and Banafsheh Nekoonam; acquisition of data: Banafsheh Nekoonam; analysis and interpretation of data: Banafsheh Nekoonam; drafting of the manuscript: Banafsheh Nekoonam; critical revision of the manuscript for important intellectual content: Azadeh Eshraghi; statistical analysis: Banafsheh Nekoonam; administrative, technical and material support: Zahra Sahraei and Mohammadreza Hajiesmaeili; study supervision: Zahra Sahraei.

Financial Disclosure: There is no financial disclosure regarding the current study.

Funding/Support: This study was supported by school of pharmacy, Shahid Beheshti University of Medical Sciences, Tehran, Iran.

\section{References}

1. Caprini JA. Thrombosis risk assessment as a guide to quality patient care. Dis Mon. 2005;51(2-3):70-8. doi: 10.1016/j.disamonth.2005.02.003. [PubMed: 15900257].

2. Sharif-Kashani B, Mohebi-Nejad A, Abooturabi SM. Estimated Prevalence of Venous Thromboembolism in Iran: Prophylaxis Still an Unmet Challenge. Tanaffos. 2015;14(1):27-33. [PubMed: 26221149].

3. Motykie GD, Zebala LP, Caprini JA, Lee CE, Arcelus JI, Reyna JJ, et al. A guide to venous thromboembolism risk factor assessment. J Thromb Thrombolysis. 2000;9(3):253-62. [PubMed:10728025].

4. Polish Working Group . A scoring system for thromboembolic risk assessment in surgery, developed by the Polish Working Group, on the basis of the scoring system developed by Joseph Caprini. Acta Angiologica. 2011;17(1):49-76.

5. Office of the Surgeon General . Section I: Deep Vein Thrombosis and Pulmonary Embolism as Major Public Health Problems. US: National Heart, Lung,Blood Institute,; 2008.

6. Ludwig KP, Simons HJ, Mone M, Barton RG, Kimball EJ. Implementation of an enoxaparin protocol for venous thromboembolism prophylaxis in obese surgical intensive care unit patients. Ann Pharmacother. 2011;45(11):1356-62. doi: 10.1345/aph.1Q313. [PubMed: 22009998].

7. Armahizer MJ, Benedict NJ, June L. FAST HUG: ICU prophylaxis. Am Soc Heal Pharm Forum. 2011:1-11. 
8. Yang SD, Liu H, Sun YP, Yang DL, Shen Y, Feng SQ, et al. Prevalence and risk factors of deep vein thrombosis in patients after spine surgery: a retrospective case-cohort study. Sci Rep. 2015;5:11834. doi: 10.1038/srep11834. [PubMed: 26135271].

9. Gaspard D, Vito K, Schorr C, Hunter K, Gerber D. Comparison of Chemical and Mechanical Prophylaxis of Venous Thromboembolism in Nonsurgical Mechanically Ventilated Patients. Thrombosis. 2015;2015:849142. doi: 10.1155/2015/849142. [PubMed: 26682067].

10. Pannucci CJ, Bailey SH, Dreszer G, Fisher Wachtman C, Zumsteg JW, Jaber RM, et al. Validation of the Caprini risk assessment model in plastic and reconstructive surgery patients. J Am Coll Surg. 2011;212(1):105-12. doi: 10.1016/j.jamcollsurg.2010.08.018. [PubMed: 21093314].

11. Zeitoun AA, Dimassi HI, El Kary DY, Akel MG. An evaluation of practice pattern for venous thromboembolism prevention in Lebanese hospitals. J Thromb Thrombolysis. 2009;28(2):192-9. doi: 10.1007/s11239-0080298-7. [PubMed: 19110614].

12. Jetha L. A drug usage review of therapeutic doses of enoxaparin at Bar- net and Chase Farm Hospitals. London: London pharmacy education and training pzifer project awards; 2007.

13. Fahimi F, Baniasadi SH, Behzadnia N, Varahram F, Ghazi Tabatabaie L. Enoxaparin utilization evaluation: An observational prospective study in medical inpatients. Iran J Pharm Res. 2010:77-82.

14. McCormick EW, Parbuoni KA, Huynh D, Morgan JA. Evaluation of Enoxaparin Dosing and Monitoring in Pediatric Patients at Children's Teaching Hospital.J Pediatr Pharmacol Ther. 2015;20(1):33-6. doi: 10.5863/1551-6776-20.1.33. [PubMed: 25859168].

15. Singh V, Gopinath K, Behzadpour A, Meera NK. Anticoagulant Utilization Evaluation in a Tertiary Care Teaching Hospital: An Observational Prospective Study in Medical in Patients. Indian JPharm Practice. 2015;8(2):61-6. doi: 10.5530/ijopp.8.2.3.

16. Khalili H, Dashti-Khavidaki S, Talasaz AH, Najmedin F, Hosseinpoor R. Anticoagulant utilization evaluation in a teaching hospital: a prospective study. J Pharm Pract. 2010;23(6):579-84. doi: 10.1177/0897190010372808. [PubMed: 21507866]. 